Apidologie, 1979, 10 (4), 301-311.

\title{
DIE CHARAKTERISIERUNG VON HONIGEN NACH IHREM GEHALT AN POLLEN UND AN STABILEN ISOTOPEN
}

\author{
La caractérisation des miels d'après leur teneur en pollen \\ et en isotopes stables
}

\author{
Hubert ZIEGLER, Anna MAURIZIO* und Willibald STICHLER** \\ Institut für Botanik und Mikrobiologie der Technischen Universität München (B.R.D.)
}

SUMMARY

THE CHARACTERIZATION OF HONEY SAMPLES ACCORDING TO THEIR CONTENT OF POLLEN AND OF STABLE ISOTOPES.

Honey samples from different, but known geographic regions were identified for their plant origin by pollen analysis and then analysed for their $\delta{ }^{13} \mathrm{C}$ - and $\delta \mathrm{D}$ - values. It was shown with one commercial sample that the isotope content can be used for the detection of falsifications. An Aloë-honey from $\mathrm{S}$ Africa proved to be the first natural honey with high $\delta{ }^{13} \mathrm{C}$ - and deuterium-values.

\section{ZUSAMMENFASSUNG}

Honigproben von verschiedenen, bekannten geographischen Herkünften wurden pollenanalytisch auf die Trachtpflanzen hin untersucht und ihre $\delta{ }^{13} \mathrm{C}$ - und $\delta \mathrm{D}$-Werte massenspektrographisch analysiert. An Hand einer im Handel befindlichen Honig-Probe wurde die Eignung der Isotopenanalyse für den Nachweis einer Verfälschung demonstriert. Eine als Alö̈-Honig deklarierte, aus S-Afrika stammende Probe erwies sich auf Grund der Pollenanalyse als richtig bezeichnet und entsprechend ihrer Herkunft von einer CAM-Pflanze als reich an ${ }^{13} \mathrm{C}$ und Deuterium. Es gibt demnach in Ausnahmefällen auch natürlicherweise "schwere " Honige.

* Liebefeld (Schweiz)

** Institut für Radiohydrometrie der Gesellschaft für Strahlen-und Umweltforschung mbH., München (B.R.D.). 


\section{I. - EINLEITUNG}

In einer früheren Arbeit wurde darauf hingewiesen, dass Nektarpflanzen gewöhnlich dem C 3-Typ der pflanzlichen Photosynthese angehören, der durch relativ geringen Anteil des stabilen Isotops ${ }^{13} \mathrm{C}$ am Gesamtkohlenstoffgehalt der organischen Substanz [ausgedrückt durch den $\delta{ }^{13} \mathrm{C}$-Wert (1)] gekennzeichnet ist. Verfälschungen mit Produkten von C 4-Pflanzen (z.B. Saccharose aus Zuckerrohr oder Stärkehydrolysaten von Mais) lassen sich deshalb in den meisten Honigsorten massenspektrographisch nachweisen (ZIEGLER et al., 1977). Zu dem gleichen Ergebnis kam unabhängig eine amerikanische Arbeitsgruppe (WHITE, 1977; DONER and WhITE, 1977: WhITE and Doner, 1978). Es wurden von uns ferner Hinweise gegeben, dass aus dem relativen Gehalt der organischen Substanz des Honigs an Deuterium (im Vergleich zum Wasserstoff, ausgedrückt durch den $\delta$ D-Wert, der analog zum $\delta{ }^{13} \mathrm{C}$-Wert gebildet wird) mit einiger Vorsicht Schlüsse auf die geographische Herkunft von Honigen. vor allem von Sortenhonigen. gezogen werden können.

Im folgenden wird an Hand weiterer, durch ihren Pollengehalt charakterisierter Honigproben aus verschiedenen Herkünften die Brauchbarkeit der geschilderten Methoden weiter erhärtet. Dabei konnte erstmals auch ein ${ }^{13} \mathrm{C}$-reicher Honig gefunden werden, der sich durch seinen ökologisch einwandfreien Pollengehalt von verfälschtem "Schwerem " Honig unterscheidet.

\section{II. - MATERIAL UND METHODEN}

Bei der Ermittlung des Pollengehaltes im Honig wurde nach Lovveaux el al. (1978) verfahren. Für die lsotopenbestimmung wurden die Proben $24 \mathrm{Std}$. bei $60^{\circ} \mathrm{C}$ getrocknet. Die weitere Präparation und die massenspcktrometrische Bestimmung erfolgte nach OsMoND et al. (1975). Zwei Werte können dann als gesichert verschieden betrachtet werden, wenn sie beim $\delta^{13} \mathrm{C}$ um mindestens $0.3 \%$, beim $\delta \mathrm{D}$ um $3 \%$ auseinander liegen.

\section{III. - ERGEBNISSE UND DISKUSSION}

Mit Ausnahme zweier gleich zu erläuternder Proben zeigen alle untersuchten Honige $\delta{ }^{13} \mathrm{C}$-Werte zwischen $-22,64 \%$ und $-26,75 \%$ (Tab. 1, 2); sie liegen demnach alle im Bereich der Pflanzen des C 3-Photosynthesetyps. Innerhalb einigermassen sortenreiner Honige sind die Schwankungen im ${ }^{13} \mathrm{C}$ - Gehalt, auch bei

(1) $\left.\delta^{\prime} \mathrm{C} \cdot 1 n_{0}\right) \cdot\left[\frac{{ }^{17} \mathrm{C} /{ }^{12} \mathrm{C} \text { Probe }}{{ }^{13} \mathrm{C} /{ }^{12} \mathrm{C} \text { Standard }}-1\right] \times 1000$ (Als Standard dient ein definierter Kalkstein.) 


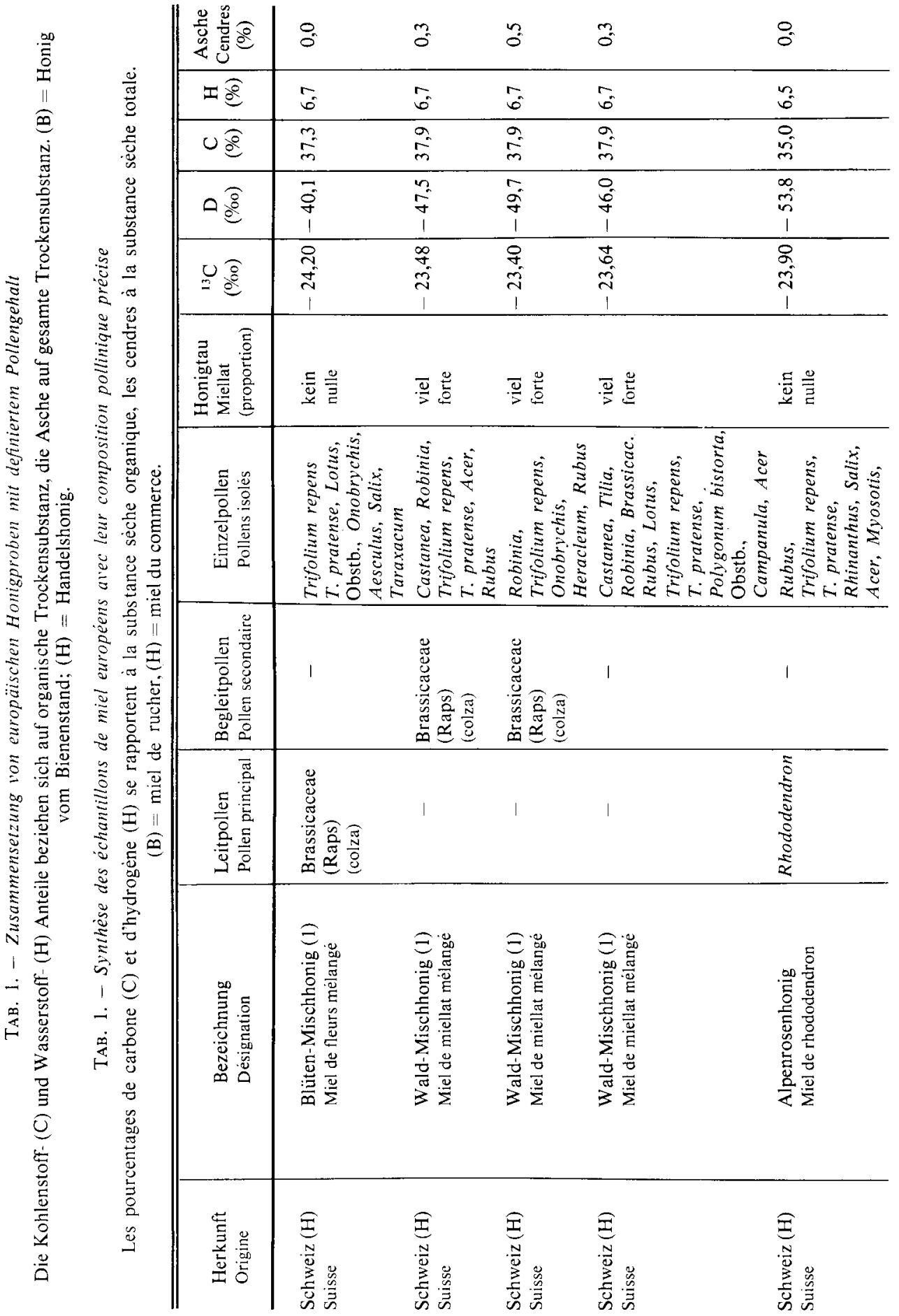




\begin{tabular}{|c|c|c|c|c|c|c|c|}
\hline 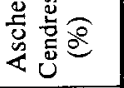 & $\tilde{o}$ & $\overrightarrow{0}$ & ? & $\stackrel{\Delta}{0}$ & $\begin{array}{l}\infty \\
0\end{array}$ & $\ddot{\sigma}$ & $\stackrel{2}{2}$ \\
\hline$x \widehat{\varrho}$ & $\overrightarrow{0}_{0}^{\circ}$ & in & 5 & S. & $g_{0}$ & $\begin{array}{l}\infty \\
0 \\
0\end{array}$ & $\begin{array}{l}\tilde{\sigma} \\
\hat{\sigma} \\
\end{array}$ \\
\hline $0 \lesssim$ & $\overrightarrow{\infty_{0}}$ & $\begin{array}{c}0 \\
\substack{0 \\
\infty \\
\infty \\
n} \\
n\end{array}$ & $\bar{i}$ & $\begin{array}{l}\dot{0} \\
\dot{m} \\
\dot{m}\end{array}$ & $\underset{m}{\stackrel{\Delta}{m}}$ & $\stackrel{\check{m}}{\bar{m}}$ & $\vec{r}$ \\
\hline ๑) & $\begin{array}{l}\frac{3}{3} \\
1\end{array}$ & $\begin{array}{c}\bar{d} \\
1\end{array}$ & $\stackrel{0}{\frac{0}{m}}$ & $\begin{array}{c}m \\
\vec{m} \\
\dot{1}\end{array}$ & $\begin{array}{c}\bar{i} \\
1 \\
1\end{array}$ & $\begin{array}{l}\infty \\
\stackrel{N}{N} \\
1\end{array}$ & $\begin{array}{l}a \\
\text { ô } \\
m \\
1\end{array}$ \\
\hline$\stackrel{0}{g}$ & $\begin{array}{l}\vec{\forall} \\
\dot{J} \\
\dot{J} \\
1\end{array}$ & $\begin{array}{c}\infty \\
+ \\
+ \\
\sim \\
1\end{array}$ & 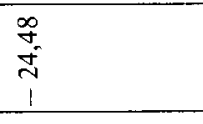 & \begin{tabular}{l} 
\& \\
\multirow{2}{*}{} \\
1
\end{tabular} & 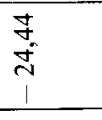 & $\begin{array}{l}\stackrel{R}{1} \\
\text { N } \\
1\end{array}$ & $\begin{array}{l}\infty \\
\infty \\
\stackrel{\sim}{1} \\
1\end{array}$ \\
\hline 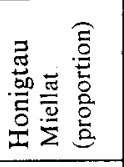 & 啮产 & 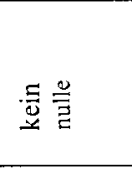 & 昰昙 & 言咅 & 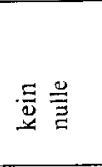 & 芽言 & 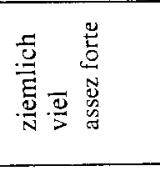 \\
\hline 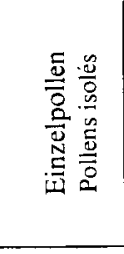 & 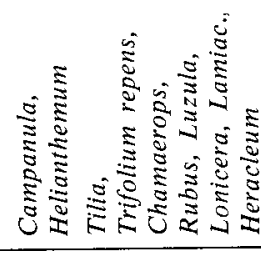 & 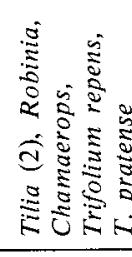 & 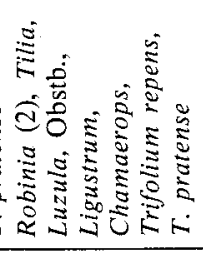 & 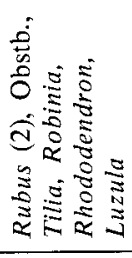 & 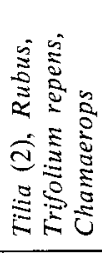 & 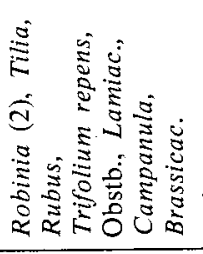 & 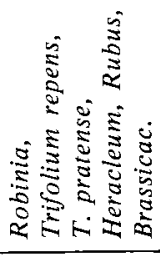 \\
\hline 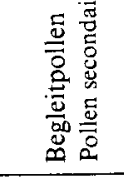 & 1 & 1 & I & 1 & 1 & 1 & 1 \\
\hline 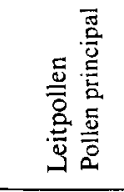 & 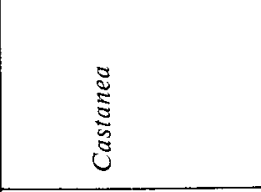 & 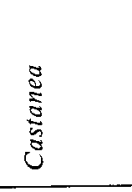 & 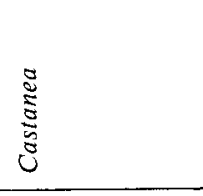 & 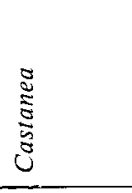 & 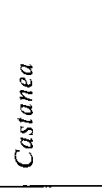 & 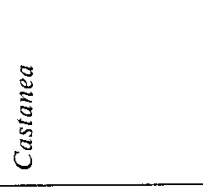 & 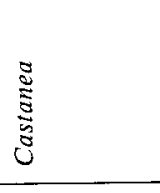 \\
\hline
\end{tabular}

\begin{tabular}{|c|c|c|c|c|c|c|}
\hline 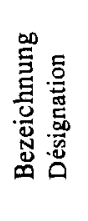 & 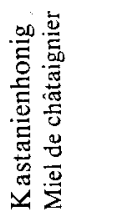 & 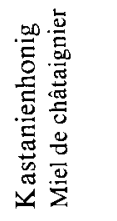 & & & & \\
\hline 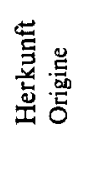 & 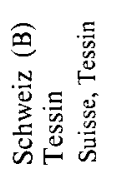 & 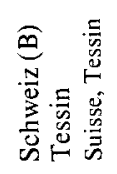 & 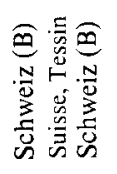 & 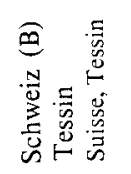 & 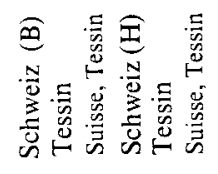 & 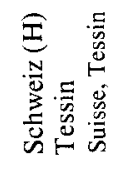 \\
\hline
\end{tabular}


$\therefore: \therefore \quad 3 \quad 3 \quad 3 \quad 3 \quad 3$

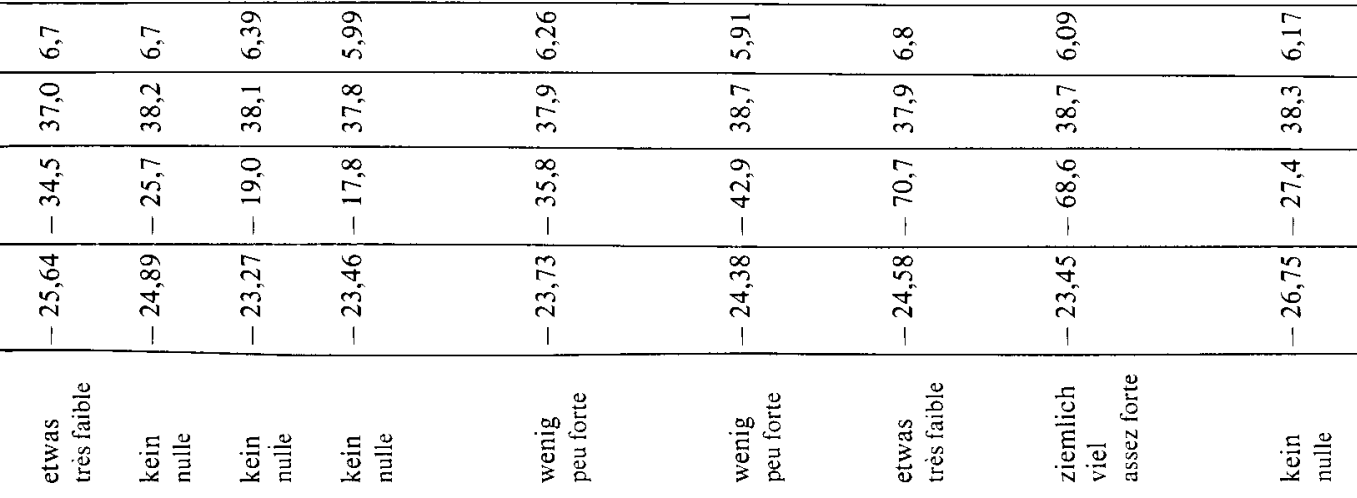

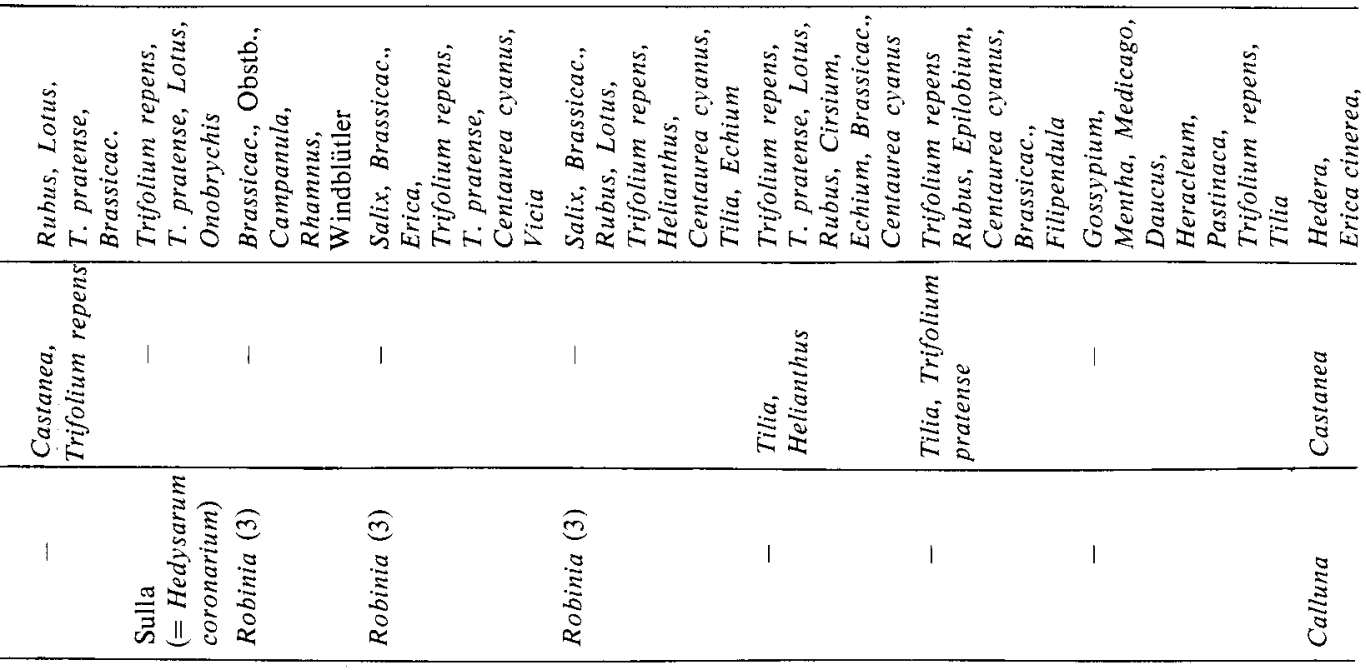

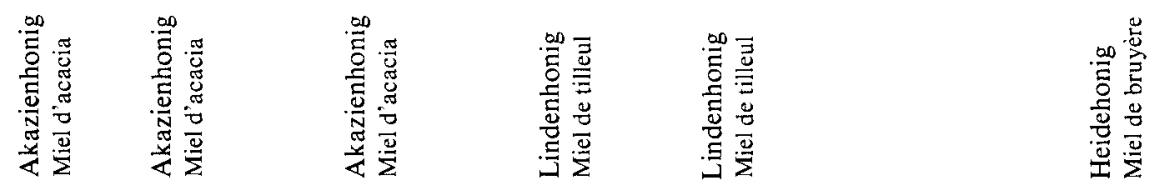

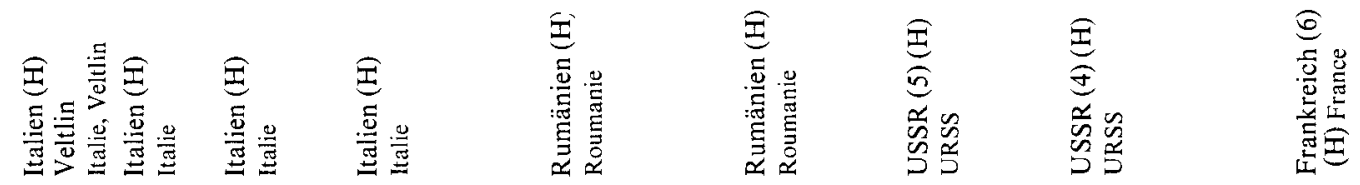


verschiedener geographischer Herkunft, sehr gering (vgl. z.B. die Schweizer Waldmischhonige, die Kastanienhonige, die Robinien-, Orangen- und Kleehonige).

Der $\delta$ D-Werte der organischen Substanz in den untersuchten Honigproben lässt wieder eine deutliche Abhängigkeit vom Klima des Herkunftsortes erkennen. Je arider der Standort der Trachtpflanzen, desto Deuterium-reicher ist der Honig. Dies wird vor allem deutlich, wenn man Honig von den gleichen Trachtpflanzen (z.B. Citrus, Robinia, Trifolium) von verschiedenen Herkünften vergleicht (Tab. 1, 2).

Die Prozentanteile von Kohlenstoff $(35,0-39,7 \%)$ und Wasserstoff $(5,70-6,90 \%)$ schwanken wieder nur relativ geringfügig. Die Aschengehalte liegen ebenfalls - wie in unseren früheren Analysen (ZIEGLER et al., 1977) - sehr niedrig, nicht selten unter der Nachweisgrenze unserer Methode.

Besonders hervorzuheben sind zwei Honigproben der Tab. 2. Die eine, angeblich von Swaziland stammende, war schon wegen ihres Pollengehaltes als zumindest mit falscher Herkunftsangabe versehen ausgewiesen. Da der Honig bei Erwärmen nach Maissirup roch, lag der Verdacht nahe, es handele sich um ein Kunstprodukt aus hydrolysierter Maisstärke, dem Pollen zugesetzt worden war. Die Bestimmung des $\delta^{13} \mathrm{C}$-Wertes bestätigt diese Vermutung vollauf : $-11,20 \%$ liegt im Bereich der Werte für Isomerose-Sirup und andere Zuckersirupe auf der Basis von Maisstärke $\left(\delta{ }^{13} \mathrm{C}\right.$-Werte zwischen- 10,21 und $-12,35 \%$; Zieglen et al., 1977). Auch der $\delta$ D-Wert liegt im Bereich der Maisprodukte.

Wie aber die Honigprobe aus Südafrika (Pretoria) in Tab. 2 erkennen lässt, müssen hohe ${ }^{13} \mathrm{C}$ - und Deuteriungehalte im Honig nicht immer eine Verfälschung der Proben bedeuten. Die Sorten- (Aloë marlothii) und Herkunftsbezeichnung dieser Probe wurde durch die Pollenanalyse bestätigt : $77 \%$ der insgesamt (von Herrn Dr. TALPAY, Bremen) gezählten 291 Pollen waren Liliaceenpollen, weitere $19 \%$ Compositen, der Rest von $4 \%$ eine Reihe anderer Taxa. Die Aloearten aber sind CAM-Pflanzen, d.h. Pflanzen mit nächtlicher $\mathrm{CO}_{2}$-Fixierung, die auf trockenen Standorten $\delta{ }^{13} \mathrm{C}$-Werte wie $\mathrm{C}$ 4-Pflanzen aufweisen und durch besonders hohe Deuteriumgehalte in der organischen Trockensubstanz ausgezeichnet sind (vgl. ZiEgler, 1979). Dementsprechend ist der Honig relativ reich an ${ }^{13} \mathrm{C}$ und Deuterium (Tab. 2).

Bei einer anderen Aloeart, Aloë eru Berger, wurden inzwischen an Nektar ${ }^{13} \mathrm{C}$ Bestimmungen durchgeführt (ZIEGLER u. LüTTGE, 1979) und Werte zwischen - 14,10 und 15,79\%o erhalten. Auch andere CAM-Pflanzen, z.B. die Asclepiadacee Hoya carnosa (L.) R.Br. $\left(\delta^{13} \mathrm{C}-12,99 \%\right.$; $\left.\delta \mathrm{D}+59,9<\right)$, die Agavacee Sansevieria trifasciata Prain cv. Laurentii N.E.Br. $\left({ }^{13} \mathrm{C}-13,76 \%\right.$; $\left.\delta \mathrm{D}+75,1 \% \mathrm{o}\right)$ und die Crassulacee Bryophyllum daigremontianum (Ham. et Perr.) Berger $\left({ }^{13} \mathrm{C}-12,40\right.$ bis - $14,68 \%$ ) zeigen nach diesen Autoren hohe ${ }^{13} \mathrm{C}$ - und Deuteriumgehalte in der organischen Substanz des Nektars (bei kultivierten Exemplaren). 


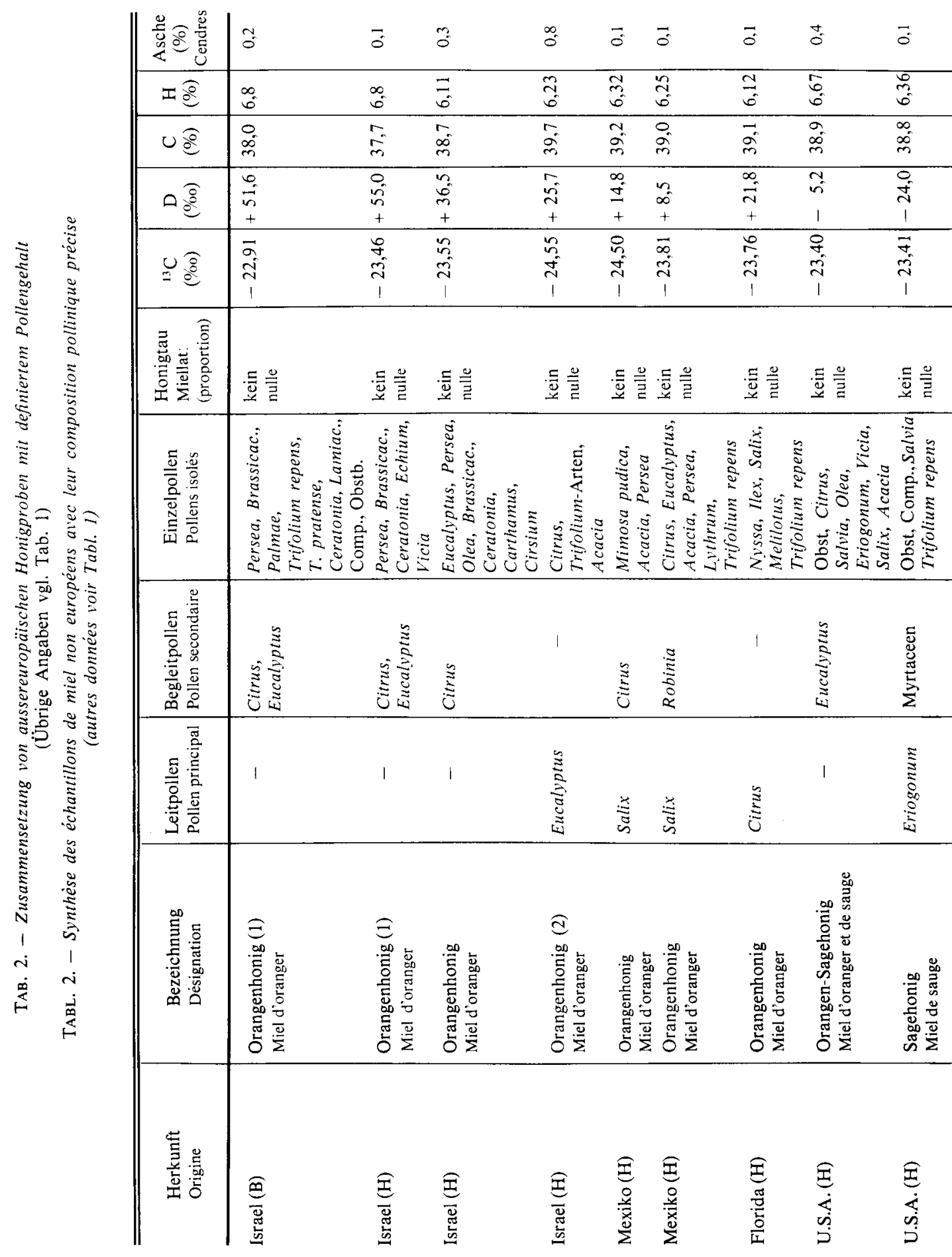




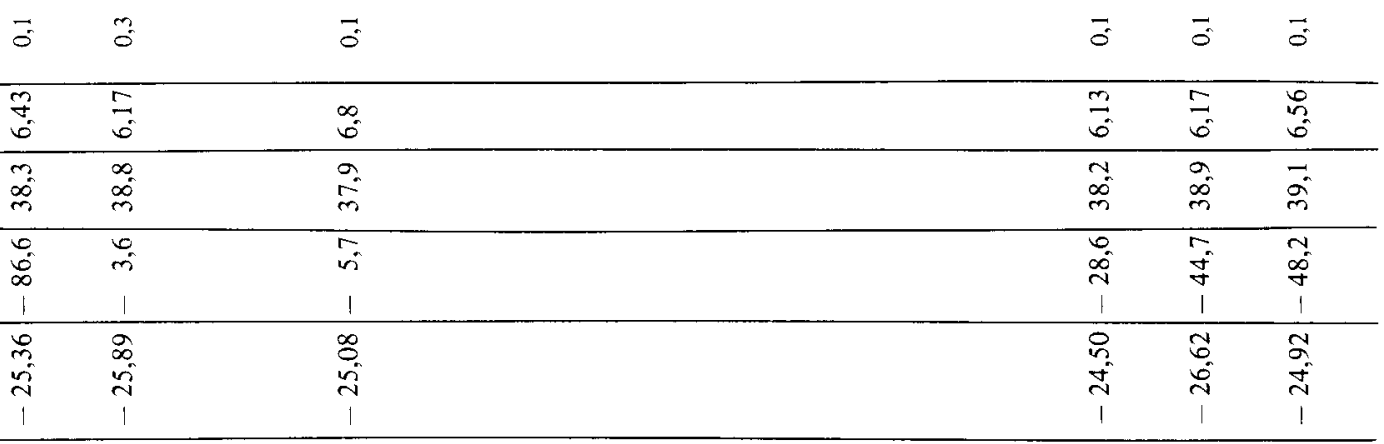

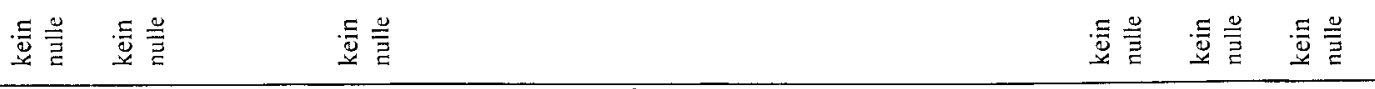

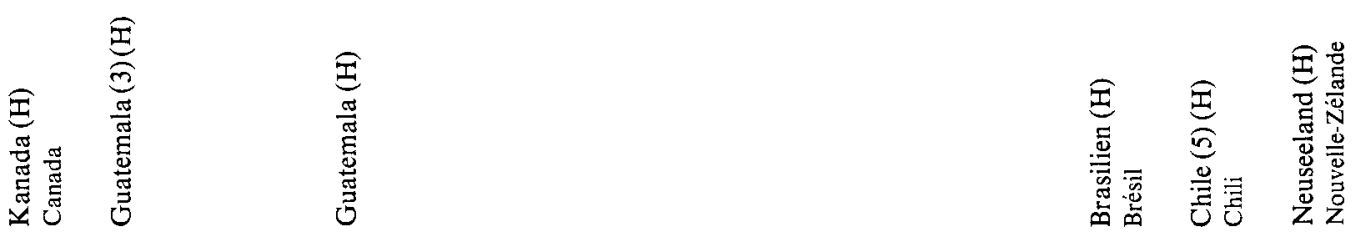




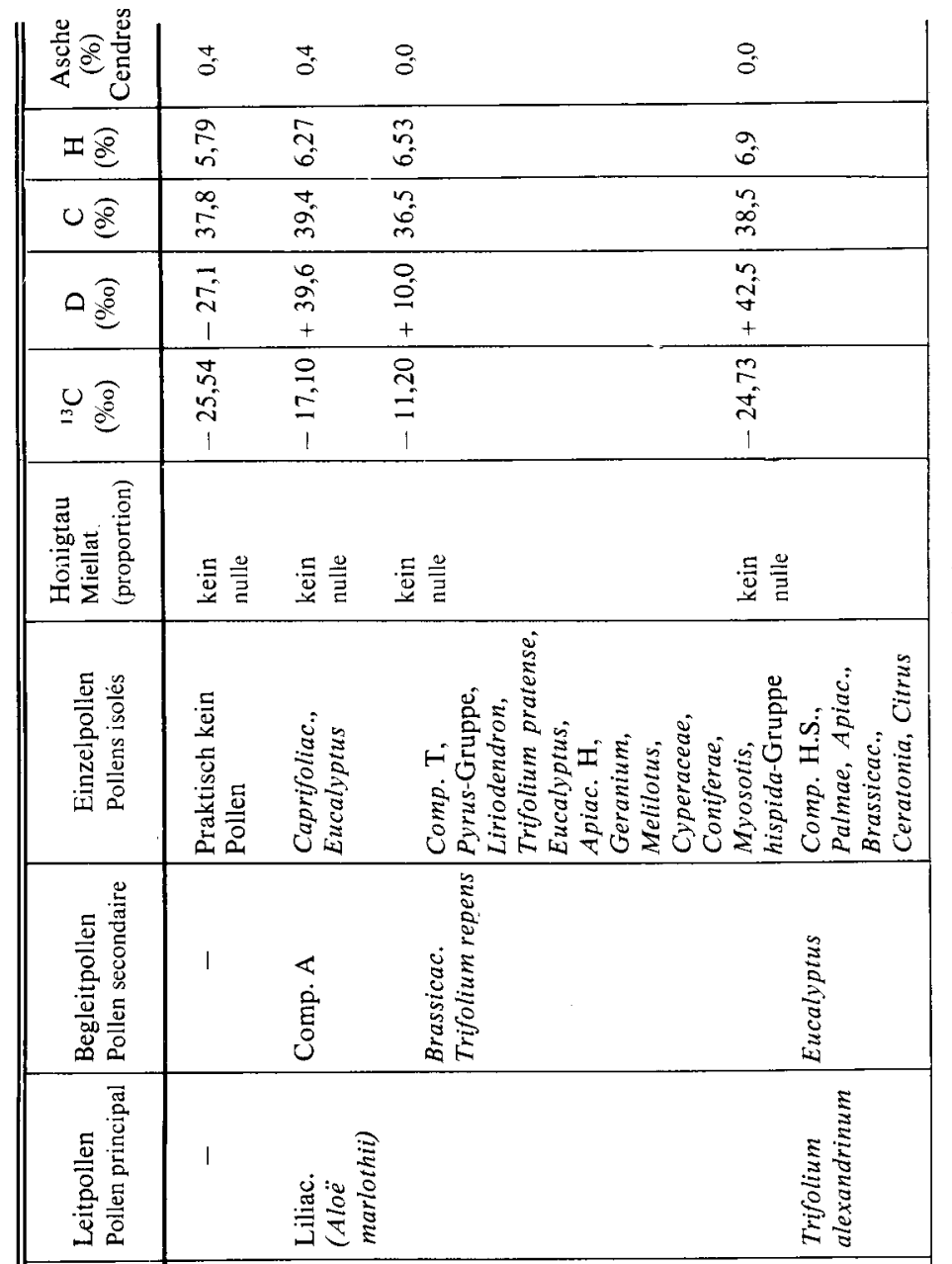

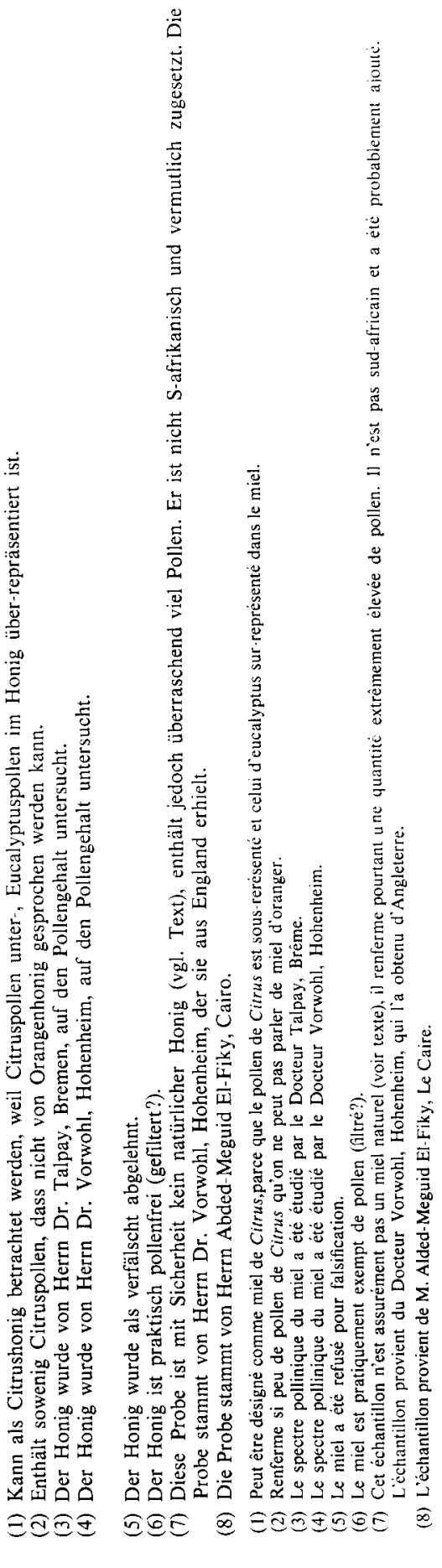


Zweifellos hätten auch Pflanzen des C 4-Typs der photosynthetischen $\mathrm{CO}_{2}$ Fixierung einen ${ }^{13} \mathrm{C}$-reichen Nektar, doch handelt es sich hier zumeist um windblütige Pflanzen. Ob es unter den C 4-Pflanzen Trachtpflanzen für die Bienen gibt, die dann wie die CAM-Pflanzen $-{ }^{13} \mathrm{C}$-reichen Honig ergäben, bleibt zu prüfen.

\section{DANK}

Wir danken den in der Arbeit genannten Kollegen für ihre Mitarbeit, Frau W. Lotz für sorgfältige technische Assistenz und der Deutschen Forschungsgemeinschaft für ihre Unterstützung.

Eingegangen im September 1979.

Reçu pour publication en septembre 1979

\section{RÉSUMÉ}

L'origine botanique d'échantillons de miel provenant de régions géographiques diverses, européennes (Suisse, Italie, France, Espagne, Roumanie, U.R.S.S.) et non européennes (Israël, Égypte, Swaziland, Afrique du Sud, U.S.A., Mexique, Guatemala, Brésil, Chili, Nouvelle-Zélande) a été étudiée par l'analyse pollinique. Le rapport ${ }^{13} \mathrm{C} /{ }^{12} \mathrm{C}$ (désigné par la valeur $\delta{ }^{13} \mathrm{C}$ ) et le rapport deutérium/hydrogène (désigné par la valeur $\delta \mathrm{D}$ ) dans la substance sèche organique ont été obtenus par spectrographie de masse. Il a déjà été montré que les falsifications du nectar ou du miel par du saccharose provenant de la canne à sucre ou par un hydrolysat d'amidon de maïs peuvent être détectées de cette manière.

L'un des échantillons testés (celui dit du Swaziland) s'est révélé falsifié de façon certaine.

Mais on a pu montrer sur un miel d'aloès d'Afrique du Sud qu'il existait aussi exceptionnellement des miels dont la substance sèche était naturellement riche en ${ }^{13} \mathrm{C}$ et en deutérium.

Mais en associant analyse pollinique et détermination isotopique on peut diagnostiquer avec sûreté les falsifications les plus courantes.

\section{LITERATUR}

Doner L. W., White J. W., 1977. - Carbon-13/Carbon-12 ratio is relatively uniform among honeys. Science 197, 891-892.

Louveaux J., Maurizio Anna, Vorwohl G., 1978. - International Commission for Bee Botany of IUBS. Methods of Melissopalynology. Bee World 59, 139-157.

Osmond C. B., Ziegler H., Stichler W., Trimborn P., 1975. - Carbon isotope discrimination in alpine succulent plants supposed to be capable of crassulacean acid metabolism (CAM). EEcologia (Berl.) 18, 209-217.

WHITE J. W., 1977. - Developing tests to detect adulteration of honey, Amer. Bee J. 117, 440-44l.

White J. W., Doner L. W., 1978. - Mass spectrometric detection of high-fructose corn sirup in honey by use of ${ }^{13} \mathrm{C} /{ }^{12} \mathrm{C}$ ratio : collaborative study. J. Assoc. Off. Anal. Chem., 61, 746-750.

ZiEgleR H., 1979. - Diskriminierung von Kohlenstoff- und Wasserstoffisotopen : Zusammenhänge mit dem Photosynthesemechanismus und den Standortbedingungen. Ber.dtsch.bot.Ges. 92, 169-184.

Ziegler H., LüTtGE U., 1979. - ${ }^{13} \mathrm{C}$ - and deuterium content in the organic material from nectar. Afrique Naturwissenchaften (in press).

Ziegler H., Stichler W., Maurizio Anna, Vorwohl G., 1977. - Die Verwendung stabiler Isotope zur Charakterisierung von Honigen, ihrer Herkunft und ihrer Verfälschung. A pidologie 8, 337-347 